\title{
An Investigation of Damage Mechanisms in Mechanobiological Models of In-Stent Restenosis
}

David R. Nolan ${ }^{1,2}$, Caitríona Lally ${ }^{1,2, *}$

1. Dept. of Mechanical and Manufacturing Engineering, School of Engineering, Trinity College Dublin, Ireland.

2. Trinity Centre for Bioengineering, Trinity Biomedical Science Institute, Trinity College Dublin, Ireland.

*Corresponding Author

+35318963159

lallyca@tcd.ie

Parsons Building, Trinity College, Dublin, Dublin 2,

Ireland

\section{Abstract}

The mechanisms behind in-stent restenosis, the re-narrowing of a stented artery, are poorly understood. However it is known that mechanical damage due to stent implantation plays a major role. This paper investigates the mechanism behind damage-induced cell proliferation using a coupled finite element and agent based model, assuming it is based on a) instantaneous loading, or b) cyclic loading. Furthermore the role of remnant endothelial cells in attenuating in-stent restenosis is examined. Results show that a cyclic damage model predicts a nonphysiological, overly proliferative response, whilst the instantaneous model demonstrates that lumen loss may be regulated by re-endothelialisation. 


\section{Acronyms}

BMS - bare metal stents

DES - drug eluting stents

EC - endothelial cell

ECM - extracellular matrix

$\mathrm{FE}$ - finite element

ISR - in-stent restenosis

ODE - ordinary differential equation

VSMC - vascular smooth muscle cell

\section{Introduction}

Cardiovascular diseases are a leading cause of death in the U.S. and Europe [1,2], with coronary atherosclerosis accounting for approximately $20 \%$ of all European cardiovascular diseases [2].

Intravascular stents are the most commonly used treatment for atherosclerosis induced vascular stenoses, with over one million procedures performed annually in the U.S. alone [3]. The most significant long-term limitation of stenting is in-stent restenosis whereby excessive migration and proliferation of vascular smooth muscle cells (VSMCs) decreases the lumen cross-sectional area post-stenting, see Figure 1 [4]. There is a strong correlation between the degree of stent arterial injury, and the subsequent decrease in lumen area due to restenotic growth [4-6]. Therefore stent designs need to be optimised to minimise the degree of vascular injury. The advent of drug eluting stents has decreased the rates of in-stent restenosis, particularly in coronary arteries [7]. Given that there are some limitations with drug-eluting stents, however, such as late thrombosis, and their limited suitability in peripheral stenting applications, the optimum stent design still remains an open question $[5,7,8]$. 
In order to develop strategies to improve stent designs and minimise in-stent restenosis, it is critical to obtain a thorough understanding of the mechanobiology of arterial tissue, i.e. how cells within arterial tissue respond to changes in their mechanical environment which may be induced by a stent. After stent deployment, quiescent, contractile VSMCs are believed to change their phenotype to proliferative, synthetic VSMCs (Figure 1A) and it is these synthetic cells which are believed to migrate to the lumen and form a restenotic lesion (Figure 1B) $[5,9]$. Damage to the surrounding extracellular matrix (ECM) has been shown to effect changes in VSMC phenotype and activation [10]. Matrix-degrading metalloproteinases (MMPs) degrade collagen in the ECM and have also been shown to regulate VSMC behaviour [11]. Variations in mechanical strain, which occurs following stenting, has also been shown to upregulate MMPs and lead to degradation of ECM [12]. Furthermore the presence of remnant endothelial cells post-stenting can limit the restenotic response [13]. Hence there is a complex, dynamic relationship between the mechanical environment induced by deployment of an intravascular stent, and the response of arterial tissue and the constituent VSMCs.

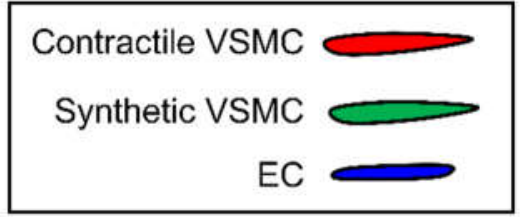

B)

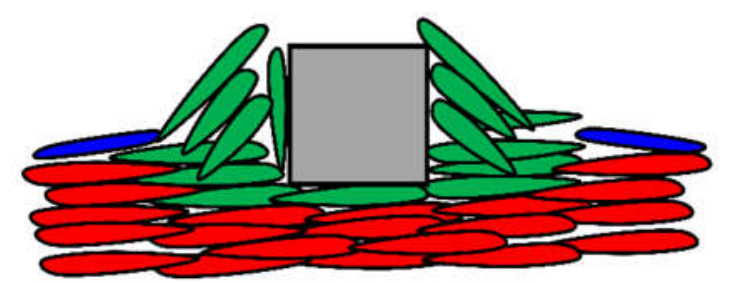

A)



C)



\section{Figure 1}

Schematic which broadly outlines the biological processes involved in bare metal ISR. A) The stent strut denudes ECs and causes the underlying normal, contractile VSMCs to change their phenotype to synthetic VSMCs. B) These synthetic VSMCs are highly proliferative and envelop the stent strut. C) ECs form a 
monolayer over the inflamed region causing the synthetic VSMCs to change back to a contractile phenotype, and ultimately ceasing VSMC proliferation and further loss of lumen area.

One approach to modelling the complex mechanobiological response of VSMCS to their mechanical environment is to use multi-scale models. To-date, such multi-scale modelling approaches have included using agent based models (ABM) or cellular automata $(\mathrm{CA})$ to model the biological vascular cell processes coupled with finite element models (FEMs) to estimate the mechanical stimuli driving the cellular processes [14-18]. Earlier work from our group used a coupled ABM-finite element (FE) model to study both intimal hyperplasia (IH) postbypass grafting [15] and stenting [14], whilst a linked group investigated the role of inflammation and IH post-stenting using a coupled CA-FE approach [16,19]. Though stent induced vascular injury has been extensively identified as the predominant stimulus for inducing and maintaining synthetic VSMC proliferation and restenotic neo-intimal growth [20], the specific mechanical stimulus analogous to this vascular injury has remained in question. Injury scores have been devised to predict neo-intimal thickness based on: deep tissue injury [9], compression of the media [21], and stretch of the internal elastic lamina [6]. Additionally, VSMCs have been shown to alter their behaviour, e.g. phenotypic changes, production of ECM and proliferation, with changes in cyclic strain [22,23]. Given the lack of consensus in the literature on the specific mechanical stimuli driving vascular injury, and ultimately the in-stent restenosis process, mechanobiological models of in-stent restenosis have been defined which are governed by many different mechanical stimuli, namely shear stress or oscillatory shear stress [24], stretch [25], von Mises stress [14] and cyclic damage accumulation [19] to name but a few.

All of these models, however, have been shown to successfully capture the key characteristics of restenosis post-stenting, albeit over somewhat different timescales and with some variability in restenosis growth patterns. The specific aim of this work was therefore to 
directly compare different damage models, particularly that of Zahedmanesh et al. [14] and Boyle et al. [19] using an ABM-FE approach for both stimuli, with a view to gaining greater insights into the mechanism of injury within stented vessels and the key role this plays in the progression of in-stent restenosis. The role of re-endothelialisation is also explored using this multi-scale mechanobiological paradigm. 


\section{Materials and Methods}

A coupled simulation environment is used to explore the role of mechanical injury and damage in ISR. This consists of two primary modules; a finite element (FE) model to compute the stress in the artery wall in response to the implantation of a stent, and an agent based model (ABM) which simulates the collective response of the cells in the artery wall to tissue damage. Figure 2 gives an overview of the two simulation modules and how they interact. The artery is idealized as a $2 \mathrm{D}$ quarter cylinder in the radial-circumferential plane with an internal radius of $1.23 \mathrm{~mm}$ and a thickness of $0.67 \mathrm{~mm}$ [26]. This simulation environment is used to model the implantation of a stent strut into the artery wall, and the resultant restenotic response. The quarter symmetry of the model decreases the computational expense of the simulation, but necessitates the use of appropriate boundary conditions (detailed below). This section outlines the important features of each of the modules in the simulation environment, and gives a procedure for model calibration. 
Finite Element Analysis Module

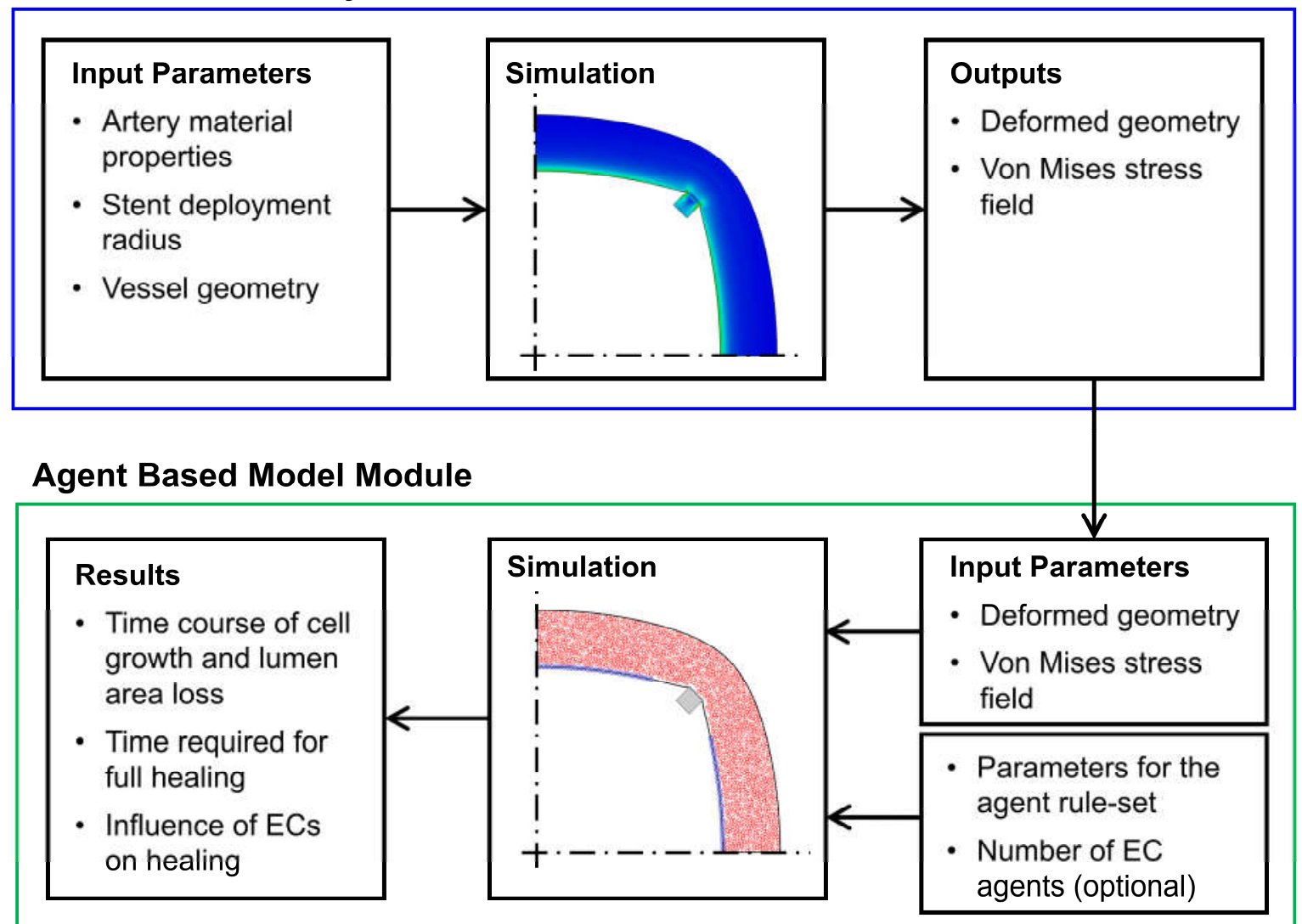

\section{Figure 2}

The simulation environment created to model ISR consists of two primary modules; a FE module and an ABM module. The simulation commences with the FE module where the mechanical response of the artery wall is determined for a scenario input by the user. The vessel wall stresses and deformed geometry are passed from the resulting FE model, and used as input parameters for the ABM module. The ABM then predicts the subsequent ISR process where the time course of VSMC proliferation and lumen area loss is calculated.

\subsection{Agent Based Model of the Artery}

An ABM is developed in Matlab (R2015b) which can simulate the collective response of cells in the artery wall to mechanical damage. Two different cell types are modelled; VSMCs and ECs. Each individual cell is modelled as a circular agent that can exist at any location within the artery wall. In general, agents may perform a number of actions; migration, proliferation and apoptosis (cell death). However in ISR VSMC proliferation is the dominant cell action and therefore migration is neglected. 
The majority of the agents in the model are VSMC agents. In the model setup phase they are seeded randomly inside the boundary of the artery wall. EC agents are seeded as a monolayer on the internal (lumen) edge of the artery wall. To simulate the mechanical damage caused by stenting, EC agents may be selectively removed from the region surrounding an implanted stent strut. Agents are seeded on the deformed geometry of the artery after stent strut implantation. This deformed geometry is computed by the FE module described in Section 2.2. Importantly, agents are not permitted to overlap one another whilst being seeded on the geometry, and throughout the simulation. This produces a contact-inhibition effect in the model.

The number of VSMC agents seeded in the model is calculated based on the density of VSMCs and the undeformed area of the artery used in the model. The undeformed area is used as the artery is in a stress-free configuration in the histological images used to determine cell density. VSMCs have an elongated spindle shape in vivo, but as an approximation are represented as circles in the model. A VSMC density of 950 cells $/ \mathrm{mm}^{2}$ and a radius of 12.96 $\mu \mathrm{m}$ are used based on values from the literature [14,27]. The total number of agents to be seeded is calculated by multiplying the VSMC density by undeformed area of the artery. A brute-force algorithm is used to seed agents, where an agent is randomly placed at a location and is accepted provided that a) it is within the boundary of the artery wall, and b) that it does not overlap an existing agent. This procedure is repeated until the total number of agents has been reached.

VSMC agents have 3 attributes or internal variables which govern their behaviour; damage $D$, MMP concentration $M$, and ECM density $E$. These internal variables are used to simulate the actions and response of VSMC agents to mechanical injury in the ISR process. They are governed by a set of ordinary differential equations (ODEs), the details of which are explained in Section 2.3. The damage variable is a function of the mechanical stress experienced by an agent at its centre point and ranges from 0 to 1 . In response to damage the 
concentration of MMP increases. This increase in MMP concentration causes a decrease in ECM density. Additionally all agents have internal variables which specify an agent's spatial position in Cartesian coordinates, and its age.

VSMC agents exist in two states; a contractile phenotype and a synthetic phenotype. In the contractile phenotypic state, VSMC agents are quiescent and non-proliferative. In the synthetic phenotype, VSMC are outside of their normal homeostatic environment and are in a proliferative state.

A VSMC agent may proliferate provided that it meets 3 criteria. Firstly, it must be of a synthetic phenotype. Secondly, a probabilistic function, based on the agent's age, must determine that the cell may proliferate. Thirdly, there must be space around the VSMC agent for a daughter cell to be produced, without overlapping any existing VSMC agents.

Cells have a doubling time, after which they proliferate and produce a daughter cell. In the $\mathrm{ABM}$ the decision whether to create a daughter cell is defined by a doubling time probability $P_{D T}$. At each time step in the ABM simulation, $P_{\mathrm{DT}}$ is calculated for each synthetic VSMC agent. Then, a number between 0 and 1 is randomly generated for each synthetic VSMC agent. If the number is less than $P_{\mathrm{DT}}$ then a daughter cell may be created if there is available space to do so.

If a VSMC agent has met the above criteria for doubling, a daughter cell may be created which is tangential to the parent cell and is the same size. The parent VSMC agent searches a full $360^{\circ}$ range at $1^{\circ}$ increments and checks whether a daughter cell may be created at that location without overlapping an existing VSMC or EC agent. Figure 3 shows a schematic of the decision-making process when determining if a daughter cell may be created around a parent cell. A list of angles at which a daughter cell may be successfully produced is determined. Then a random number generator is used to pick a single location from the list at which the daughter cell is ultimately created. This daughter cell inherits the damage $D$, MMP 
concentration $M$, and ECM density $E$ of its parent cell. The age of both the parent and daughter cells are set to 0 .

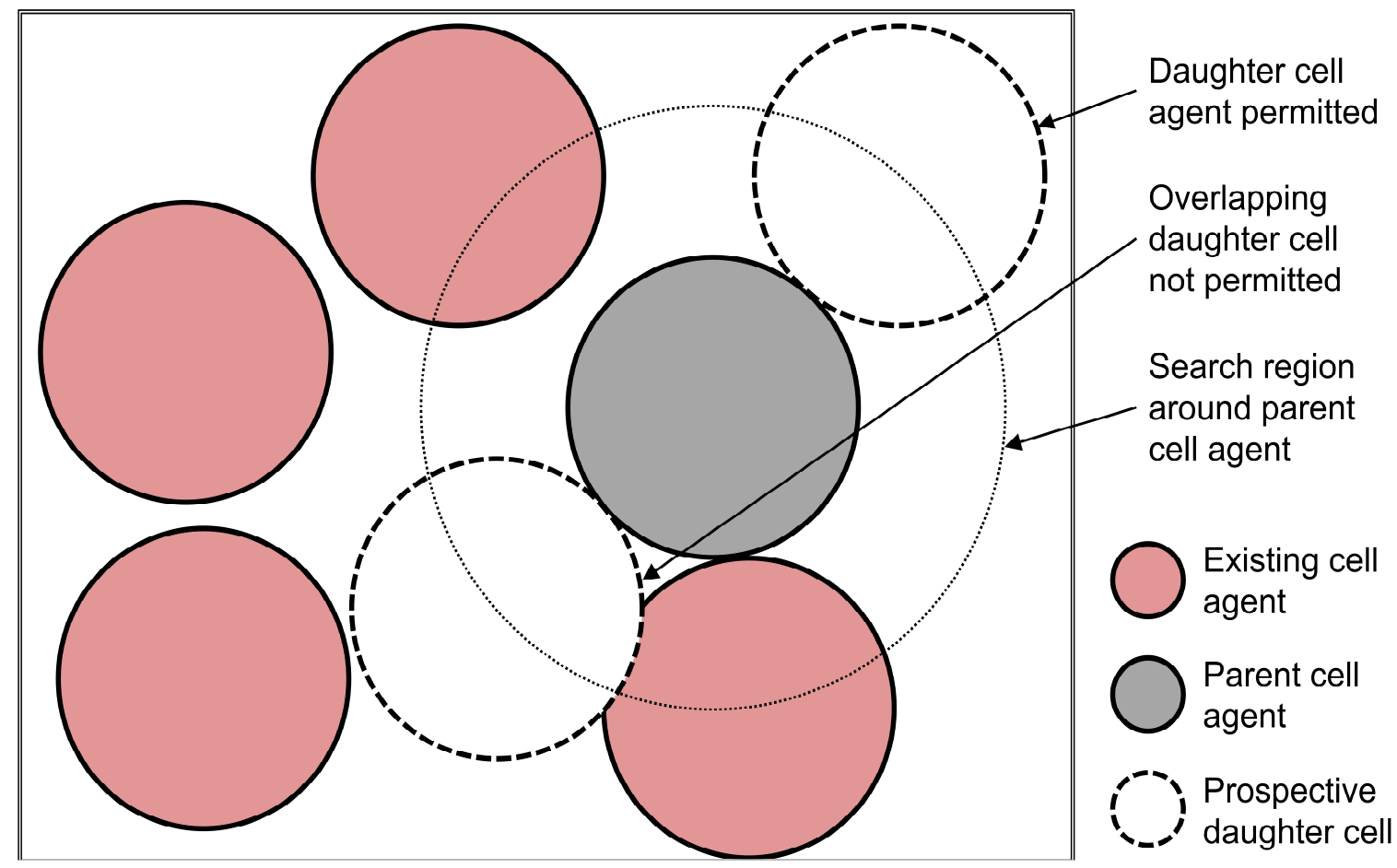

\section{Figure 3}

Schematic demonstrating how the ABM determines whether a daughter VSMC agent may be produced or not. The parent VSMC agent searches the region surrounding it in $1^{\circ}$ increments, each time checking whether a daughter VSMC agent may be produced at that location without overlapping an existing cell agent. All of the angles at which a daughter cell may be produced are determined, and a daughter cell is placed at a location picked randomly from the viable set of angles.

EC agents may be included in the ABM if desired. They are idealized as circles and seeded as a monolayer on the lumen of the artery. The radius of the EC agents is determined from histological images and set as $17.87 \mu \mathrm{m}[14,28]$. To simulate the effect of EC denudation after mechanical injury cause by stent implantation, EC agents are removed from the region both sides of the stent strut. ECs proliferate over time as part of the healing process with a fixed doubling time of 92 hours [14]. EC agents with only one neighbour proliferate into the available space whilst still maintaining a monolayer on the lumen surface. As the healing continues the 
two EC fronts progress from both sides of the stent strut and ultimately join, ceasing EC proliferation. Proximity of a VSMC agent to an EC agent changes a synthetic phenotype VSMC to a contractile phenotype. This simulates the quiescent effect of NO released by ECs. If the distance between the centre of an EC and VSMC agent is less than $60 \mu \mathrm{m}$ then the VSMC agent has a contractile phenotype [29]. Thus, as the EC agents proliferate as part of the healing process more VSMC agents will come into their domain of influence and change their phenotype. This has the net effect of slowing down and localising the proliferation of synthetic VSMC agents. In this study, the role of the number of ECs present after stenting on the resultant restenotic lumen area loss is parametrically investigated.

Finally, periodic boundary conditions must be used to correctly simulate the quarter symmetry employed in the model. The model is $\pi / 2$ periodic meaning that the west and south edges of the model are coupled. If a cell were to migrate through the west edge of the model, it should appear at the south edge at the same radial position with respect to the centre point. Migration is not used in the present model, however periodic boundary conditions must be used when calculating whether there is sufficient space in the model for a daughter VSMC agent during the mitosis phase. Phantom VSMC agents are created at the west and south edges to simulate the presence of VSMC agents located $\pi / 2$ away from that edge. These phantom VSMC agents are removed after the mitosis algorithm has completed.

A key aspect of the ABM-FE model presented here is the evolution of the internal variables $D, M$, and $E$ of each agent. In the present study two approaches are taken to their calculation, and these are described in detail in Section 2.3. A flowchart which outlines the algorithm that determines VSMC agent behaviour is given in the supplementary material that accompanies the online version of this article. 


\subsection{Finite Element Model of the Artery}

A FE model is generated of the quarter artery geometry described above and solved using the commercial FE code Abaqus (v6.14, DS Simulia). Figure 4 shows a schematic of the geometry, loading, and boundary conditions used for the FE model. The artery is partitioned into two concentric sections for the medial and adventitial layers at a ratio of 5:3 respectively [26]. Note that the intimal layer is incorporated into the media for the FE model. The material behaviour of the artery tissue is modelled using an Ogden incompressible hyperelastic constitutive equation. Separate material parameters are used for each of the layers, calibrated from the uniaxial mechanical behaviour of each layer in the circumferential direction [14]. Plane strain elements are used to simulate the out-of-plane behaviour, together with a hybrid pressuredisplacement element formulation for the efficient implementation of material incompressibility. A mesh convergence study showed that an element of approximate side length $3 \mu \mathrm{m}$ is required. To correctly enforce the symmetry boundary conditions, the south edge of the artery geometry is constrained in the $y$-direction and the west edge in the $x$ direction. Contact between the stent and artery wall is modelled using a surface to surface contact algorithm.

This FE model is used to perform a simple 2D simulation of stent implantation. Additionally, it is used to simulate balloon angioplasty - the results of which are used to calibrate the ABM (see Section 2.4 for details). 


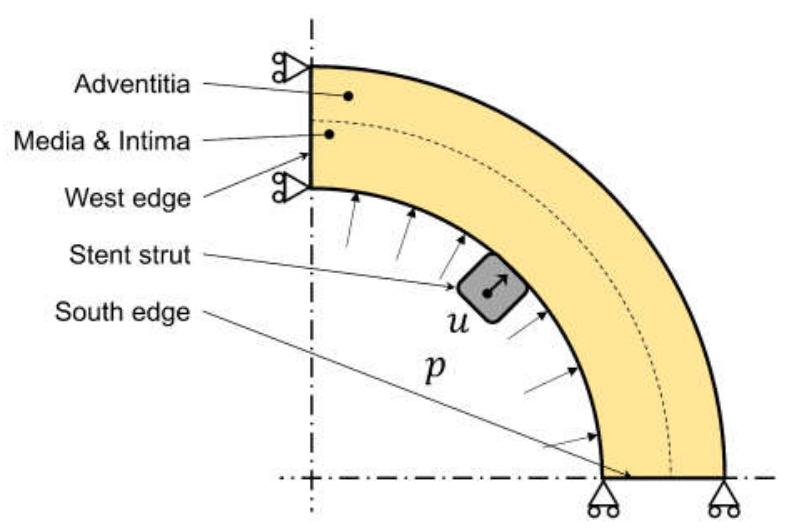

\section{Figure 4}

Schematic outlining the loads and boundary conditions used for the FE model simulations. A quarter artery is simulated with rolling boundary conditions on the edges. The artery is subjected to a uniform pressure $p$ on its internal lumen face. A stent strut is displaced into the artery wall. Separate material properties are used to simulate the adventitia and combined media and intima layers.

The first step in simulating stent implantation is inflation of the artery to a systolic pressure of $120 \mathrm{mmHg}$. Next, stent implantation is simulated by displacing a square strut of side $170 \mu \mathrm{m}$ with a fillet of $20 \mu \mathrm{m}$ into the artery wall until the stent strut is 1.1 times the radius of the artery at systolic pressure. The mean cyclic stress between the systolic and diastolic phases of the cardiac cycle, whilst the artery is stented, is required for the CDM described in Section 2.3. The systolic configuration has just been computed, and the diastolic configuration is computed by reducing the internal pressure to $80 \mathrm{mmHg}$. The mean von Mises stress at an integration point between these two configurations is the mean stress used in the CDM.

Balloon angioplasty is simulated by displacing the artery to 1.4 times its radius at systole. For the CDM, the first cycle of damage is calculated using the stress in this balloon expanded configuration. The remaining cycles are calculated at the unexpanded mean systolic and diastolic von Mises stresses. 


\subsection{Damage Stimuli for VSMC Agents}

To simulate mechanical injury to the artery wall, a damage variable is introduced. This damage variable acts as a stimulus for VSMC agents in the ABM described in Section 2.1. Two published models for the calculation of damage experienced by VSMC agents are compared; an instantaneous damage model (IDM) [14], and a cyclic damage model (CDM) [19].

The IDM and CDM are both a function of von Mises stress. The spatial distribution of von Mises stress in the artery wall is computed in the FE model outlined in Section 2.2. The von Mises stress experienced by an agent is calculated by determining the element integration point closest to its centre point and assigning the stress at that integration point to the agent. As the characteristic size of the elements is smaller than that of the agents, there is little interpolation in this technique. Once the von Mises stress for an agent is computed, the damage may then be calculated.

\subsubsection{Instantaneous Damage Model}

The IDM assumes that damage to VSMCs occurs instantly upon injury, but that this damage constantly decreases as the VSMCs commence the process of healing. The magnitude of initial damage $D_{0}$ assigned to the VSMC agent is calculated using a damage function formulated by Zahedmanesh et al. [14] which is based on experimental data of the mechanical failure of coronary artery tissue presented by Holzapfel et al. [26].

$$
D_{0}=\frac{\beta}{\beta+\exp \left[-\beta\left(\sigma_{\text {mises }}-\sigma_{0}\right)\right]}
$$

where $\beta$ and $\sigma_{0}$ are equation parameters and $\sigma_{\text {mises }}$ is the von Mises stress in the tissue. This produces a sigmoid shaped curve where for small values of stress there is zero damage, as the stress increases the damage instigates and ultimately the upper limit of one for the damage is calculated. 
The initial damage is calculated for all VSMC agents in the ABM. A set of coupled ordinary differential equations (ODEs) define the evolution with time $t$ of the internal variables of a VSMC agent; damage $D$, MMP concentration $M$, and ECM density $E$ [14].

$$
\begin{gathered}
\frac{d D}{d t}=-k_{\mathrm{deg}}^{D} M, \\
\frac{d M}{d t}=f(D)-k_{\mathrm{deg}}^{M} M, \\
\frac{d E}{d t}=k_{\mathrm{gen}}^{E}-k_{\mathrm{deg}}^{E} M,
\end{gathered}
$$

where $k$ is an equation parameter for the ODEs whose superscript denotes the internal variable to which it pertains, and the subscript indicates whether it has a generating or degrading effect on its internal variable. The rate at which MMP is created is determined by the sigmoid-shaped function $f(D)[14,25,30]$,

$$
f(D)=\frac{\kappa_{a}}{1+\kappa_{b} \exp \left(-\kappa_{c} D\right)}
$$

where the three $\kappa$ parameters are taken from Zahedmanesh et al. [14] $\left(\kappa_{a}=96.1 \times 10^{-6}, \kappa_{b}=\right.$ $\left.3468, \kappa_{c}=16.8\right)$. The coupled ODEs given in (2), (3), and (4) are solved using a forward Euler method and a time step of 1 hour, which is sufficiently small to produce a stable solution. The ODEs are solved for each agent using initial conditions. The initial condition for damage $D_{0}$ is calculated using Equation (1), the initial MMP concentration $M_{0}$ is zero, and the initial ECM density $E_{0}$ is $310 \mathrm{pg} /$ agent [14,31].

A cumulative Gaussian distribution function is introduced to simulate a cell's decision on whether to proliferate or not. The probability of a VSMC agent doubling $P_{\mathrm{DT}}$ for the IDM is defined by the equation, 


$$
P_{\mathrm{DT}}\left(t_{\mathrm{age}}\right)=\frac{1}{2}\left(1+\operatorname{erf}\left[\frac{t_{\mathrm{age}}-\mu_{\mathrm{DT}}}{\sqrt{2 \sigma_{\mathrm{DT}}^{2}}}\right]\right)
$$

where $t_{\text {age }}$ is the age of the VSMC agent, $\mu_{\mathrm{DT}}$ is the mean doubling time of a VSMC with a standard deviation of $\sigma_{\mathrm{DT}}$. This is a sigmoid shaped probability function where new VSMC agents have a 0 probability of doubling, and as the agents get older the probability gradually and smoothly starts to increase until it is ultimately equal to 1 .

In the IDM, the density of ECM at a VSMC agent is used to determine phenotype; if a cell is below a critical threshold $E_{\text {crit }}$ of $310 \mathrm{pg} /$ agent then it has a synthetic phenotype, however if it is above the critical threshold then it is contractile.

A)

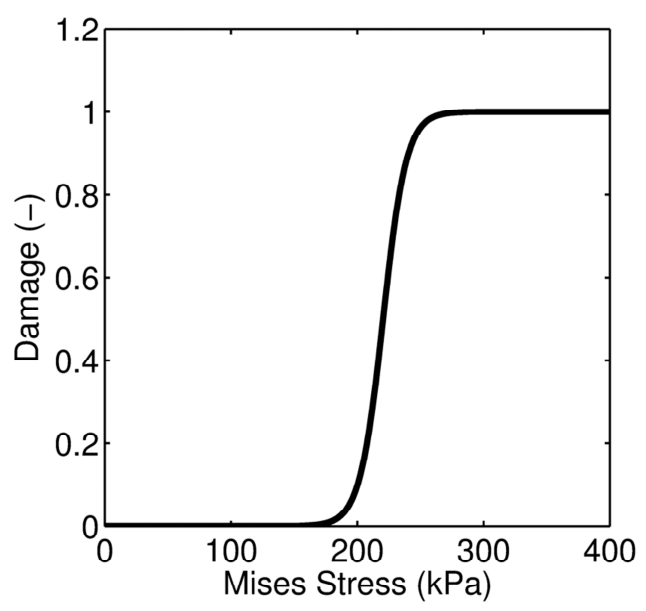

B)



\section{Figure 5}

A) Von Mises stress - damage relationship given in Equation (1) for the IDM. Note that at low values of stress there is zero damage but that as the stress increases the damage rapidly increases at a stress of around $200 \mathrm{kPa}$, with a full damage value of one given to tissue stressed at $300 \mathrm{kPa}$ and above. B) A $S-N$ curve for arterial tissue adapted from [19]. This plot gives the number of cycles to failure of an artery, $N_{f}$, when subjected to a cyclic mean stress of $\sigma_{m}$. This data is used to calibrate the parameter $\alpha$ in Equation (7) and set the fatigue limit $\sigma_{0}$ and failure strength $\sigma_{f}$. 


\subsubsection{Cyclic Damage Model}

The CDM is outlined in Boyle et al. [19] and assumes that cyclic loading at high stress is the primary mechanism behind VSMC damage. In this model damage accumulates with time, as the number of loading cycles experienced by a VSMC increases. The CDM is based on a loglinear relationship for damage due to fatigue, and Miner's rule for the number of loading cycles $N$ to failure for a material, resulting in the damage rate per cycle $\dot{D}$ equation,

$$
\begin{aligned}
\dot{D} & =10^{-\left(\sigma_{f}-\sigma_{\text {mises }}\right) / \alpha}, \\
& =0 \quad \sigma_{\text {mises }} \leq \sigma_{0} \\
& =1 \quad \sigma_{\text {mises }}>\sigma_{f}
\end{aligned}
$$

where $\sigma_{0}$ is the fatigue limit (the value of stress at which damage starts to accumulate), and $\sigma_{f}$ is the failure strength (the value of stress where one cycle results in a damage rate $\dot{D}=1$, essentially the uniaxial strength of the material, here $\sigma_{f}=1417 \mathrm{kPa}$ ), and $\alpha$ is a material constant $(\alpha=-187)$, and $\sigma_{0}$ is the endurance limit of $90 \mathrm{kPa}$. Figure 5B shows a plot of the number of cycles to failure $N_{f}$ at a mean cyclic stress $\sigma_{m}$ for porcine coronary artery tissue. Note that at stresses below $90 \mathrm{kPa}$, this arterial tissue can withstand an infinite number of cycles.

The evolution of the VSMC agent internal variables is governed by a set of coupled ODEs. Two additional internal variables are included in this model, the VSMC phenotype $\phi$ varies between zero (fully contractile phenotype) and one (full synthetic phenotype), and a growth stimulus $G$.

$$
\begin{aligned}
& \frac{d D}{d t}=f \cdot \dot{D}-c_{d e g} M, \\
& \frac{d M}{d t}=c_{g e n}^{M} D-c_{d e g} M
\end{aligned}
$$




$$
\begin{gathered}
\frac{d E}{d t}=c_{g e n}^{E} \phi-c_{d e g} M, \\
\frac{d \phi}{d t}=1-E-\phi \\
\frac{d G}{d t}=c_{g e n}^{G} D-c_{d e g}^{G} G
\end{gathered}
$$

where $c$ are equation constants whose superscript denotes the internal variable to which it pertains, and the subscript indicates whether it has a generating or degrading effect on its internal variable. The equations are solved using a forward Euler method using a time increment of 1 hour and initial conditions; $D_{0}=0, M_{0}=0, E_{0}=1, \phi_{0}=0$, and $G_{0}=0$. Damage in the material is accumulated on the basis of the number of cycles it has experienced $N$, and the frequency $f$ is the number of cycles per hour experienced by the artery. In this model that is set to 3,600 cycles/hour $(1 \mathrm{~Hz})$. In contrast the original model by Boyle et al. used a frequency of 1 cycle/day.

Finally, the probability of a VSMC agent proliferating in the CDM is defined by the equation,

$$
P_{D T}=p_{\mathrm{vsmc}} G \phi \Delta t
$$

where $\Delta t$ is the time increment used in the forward Euler solution of the ODEs, $p_{\mathrm{vsmc}}$ is the intrinsic maximum proliferation rate of a VSMC cell. So, if a VSMC agent is fully synthetic and has a relative growth of 1 , then the probability of a cell doubling is at its maximum. 


\section{Table 1}

Parameters used for the instantaneous damage model (IDM) equations (1)-(4).

\begin{tabular}{|l|l|l|l|l|l|}
\hline $\begin{array}{l}k_{\text {deg }}^{D} \\
\left(\text { cell } \mathrm{pg}^{-1} \mathrm{hr}^{-1}\right)\end{array}$ & $\begin{array}{l}k_{\text {deg }}^{M} \\
\left(\mathrm{hr}^{-1}\right)\end{array}$ & $\begin{array}{l}k_{\text {gen }}^{E} \\
\left(\mathrm{pg} \mathrm{cell}^{-1} \mathrm{hr}^{-1}\right)\end{array}$ & $\begin{array}{l}k_{\text {deg }}^{E} \\
\left(\mathrm{hr}^{-1}\right)\end{array}$ & $\beta$ & $\begin{array}{l}\sigma_{0} \\
(\mathrm{kPa})\end{array}$ \\
\hline 0.661 & 0.018 & 0.307 & 410 & 0.1087 & 200 \\
\hline
\end{tabular}

Table 2

Parameters for the cyclic damage model (CDM) equations (8)-(13).

\begin{tabular}{|c|c|c|c|c|c|}
\hline $\begin{array}{l}c_{\text {deg }} \\
\left(\text { cell } \mathrm{pg}^{-1} \mathrm{hr}^{-1}\right)\end{array}$ & $\begin{array}{l}c_{\text {gen }}^{M} \\
\left(\text { pg cell }{ }^{-1} \mathrm{hr}^{-1}\right)\end{array}$ & $\begin{array}{l}c_{\text {gen }}^{E} \\
\left(\text { pg cell }{ }^{-1} \mathrm{hr}^{-1}\right)\end{array}$ & $\begin{array}{l}c_{g e n}^{G} \\
\left(\mathrm{hr}^{-1}\right)\end{array}$ & $\begin{array}{l}c_{g e n}^{G} \\
\left(\mathrm{hr}^{-1}\right)\end{array}$ & $p_{\text {vsmc }}$ \\
\hline 0.0271 & 0.0017 & 0.001 & 0.0033 & 0.00045 & 0.029 \\
\hline
\end{tabular}




\subsection{Calibration of Damage Stimuli through Simulation of Balloon}

\section{Angioplasty}

The damage and proliferation response of VSMC agents used in the simulation of ISR is first calibrated by performing a simulation of balloon angioplasty. The parameters of the damage model ODEs described in Section 2.3 are tuned to match the predicted neointimal growth observed in clinical data published by Schwartz et al. [20]. The IDM and CDM are both adapted from existing models in the literature and the majority of the parameters for these models are taken directly from their respective papers; Zahedmanesh et al. [14] and Boyle et al. [19]. A full list of the parameters used in the paper are given in 
Table 1 and Table 2.

Schwartz et al. [20] measured the neointimal cross sectional area in a coronary artery at 4 time points after balloon angioplasty. Together with the initial lumen area, the percentage loss of lumen area over time was plotted. Initially there is large proliferation of VSMCs leading to a sharp loss in lumen area. At approximately 85 days post-angioplasty this lumen loss quickly decreases and up to 360 days post procedure, there is almost no further lumen loss.

The simulations assume that all ECs have been denuded by the procedure and thus only VSMC agents are modelled. The mean doubling time in the IDM, $\mu_{\mathrm{DT}}$ in Equation (6), is calibrated to reproduce the initial growth phase of the neointimal tissue, while the parameter controlling the generation of ECM, $k_{\text {gen }}^{E}$ in Equation (4), is calibrated to reach the point at which proliferation ceases. Likewise, in the CDM the maximum probability of cell doubling, $p_{\mathrm{vsmc}}$ in Equation (13), and the growth rate constant, $c_{\text {gen }}^{G}$ in Equation (12), are calibrated to match the lumen loss clinical data. 


\section{Results}

\subsection{Balloon Angioplasty Calibration Results}

Balloon angioplasty is simulated using the coupled ABM-FE environment described in Section 2 to calibrate the parameters for the damage stimuli equations. Figure 6 plots the predicted percentage lumen area loss over the days post angioplasty for both the IDM and CDM presented in Section 2.3. Additionally points are added to the plot indicating the percentage lumen loss measured in vivo by Schwartz et al. [20] at 4 time points post angioplasty. Simulations using both the IDM and CDM are capable of reproducing the neointimal growth behaviour after balloon angioplasty, thus calibrating the VSMC damage model ODEs.

$$
\begin{aligned}
& - \text { IDM } \\
& \text { - } \text { CDM } \\
& \text { - Schwartz (1996) }
\end{aligned}
$$

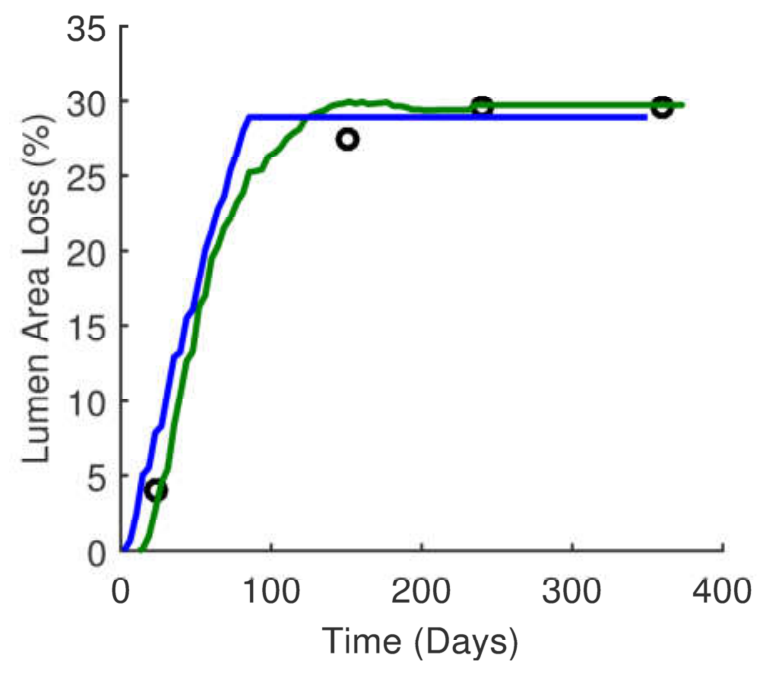

\section{Figure 6}

Plot of percentage lumen area loss versus the number of days post procedure of balloon angioplasty. The circle symbols indicate the clinical measurements outlined in Schwartz et al. [20]. 


\subsection{Predictions of In-Stent Restenosis}

\subsubsection{Predictions using VSMC Agents Only}

The proliferation of synthetic VSMCs post stenting is simulated using the IDM and CDM as damage stimuli for the VSMC agents. ECs are not included in this scenario. Figure 7A and B present the results of the simulations at 62 days using the IDM and CDM respectively. Note that the CDM predicts a large amount of lumen loss. This can be clearly seen in Figure $7 \mathrm{C}$, which plots the percentage lumen area loss with time. The CDM initially predicts small lumen loss, however the rate of loss increases and at 81 days the vessel is totally occluded with a lumen area loss of $100 \%$. In contrast, the IDM predicts a steady proliferation which ceases to increase after reaching a lumen area loss of $26 \%$.

A)

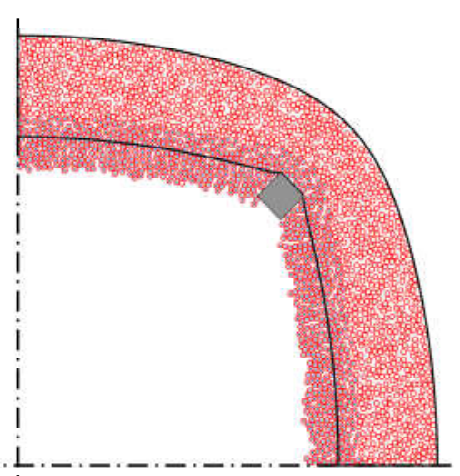

B)

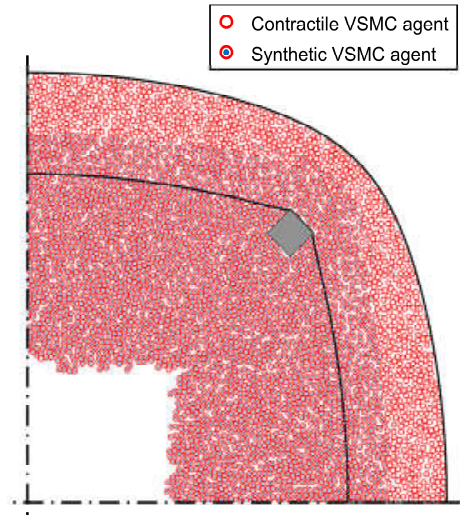

C)

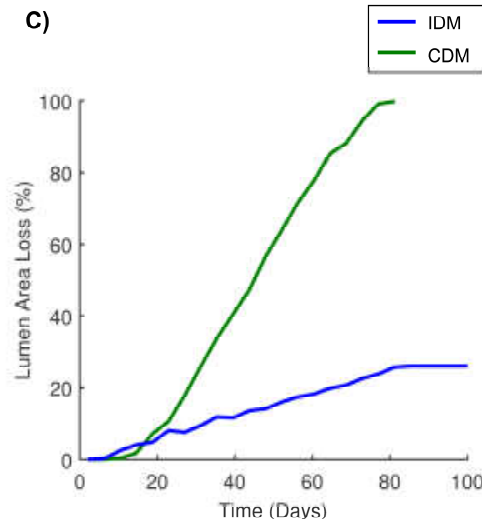

\section{Figure 7}

Result for the ABM module of the simulation showing the predicted proliferation of synthetic VSMC agents at 62 days using A) the IDM, and B) the CDM. EC agents were not included in these simulations. C) Plot showing the percentage loss of lumen are over time predicted by the IDM and CDM.

\subsubsection{Predictions Including EC Agents}

The role of ECs on the extent of lumen area loss is investigated by parametrically removing EC agents to both sides of the stent and performing a simulation. The responses predicted by the IDM and CDM are examined. Figure 8A shows the result from the ABM using the IDM of 
the proliferation of VSMCs around a stent strut at 58 days with a total of $30 \mathrm{EC}$ agents initially removed. Note the full re-endothelialisation of the lumen and the band of synthetic VSMC agents beneath the healed region. Figure $8 \mathrm{~B}$ shows the result from the $\mathrm{ABM}$ module using the IDM at 116 days with a total of 60 ECs initially removed. Again, note there is full reendothelialisation and the greater extent of lumen area loss in this case. Figure $8 \mathrm{C}$ plots the percentage lumen area loss predicted by the IDM for a given number of removed EC agents. In each scenario, the lumen area loss reaches a plateau when the lumen is fully reendothelialised.
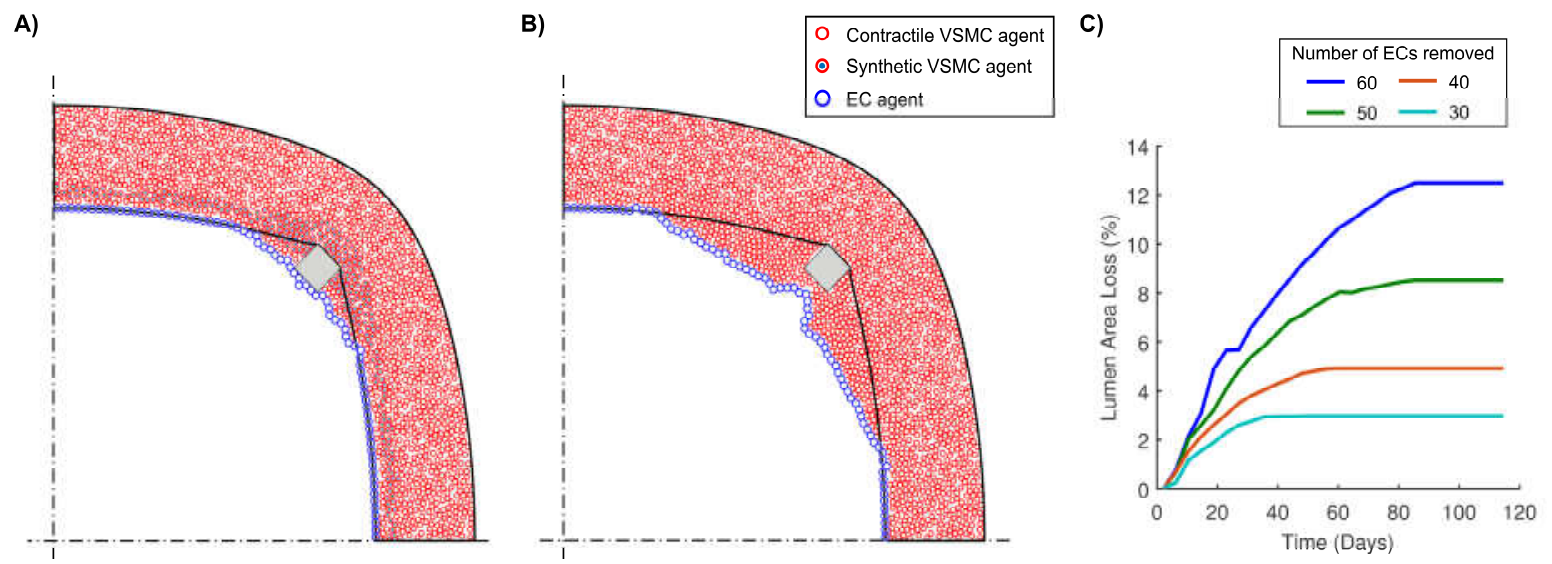

\section{Figure 8}

Result from the ABM module of the simulation using the $I D M$ showing the predictions at; A) 58 days with a total of $30 \mathrm{EC}$ agents initially removed, and B) 116 days with $60 \mathrm{EC}$ agents initially removed. C) Plot of the predicted lumen area loss over time as a function of the number of $\mathrm{EC}$ agents that are removed from the lumen.

The results for the same parametric study of EC agent removal, this time using the CDM, are presented in Figure 9. Figure 9A shows the results of the ABM at 58 days with 30 EC agents initially removed. Note the large numbers of synthetic VSMC agents which have proliferated into the lumen and the incomplete formation of an EC monolayer. Figure 9B shows the result of the $\mathrm{ABM}$ at 58 days with $60 \mathrm{EC}$ agents initially removed. Once again, note the even greater proliferation of synthetic VSMC agents into the lumen and incomplete re- 
endothelialisation. Figure 9C plots the percentage lumen area loss over time for a range of initially removed EC agents. In all cases, the lumen is totally occluded by 100 days, the only difference between each scenario being the rate at which this occurs.

A)

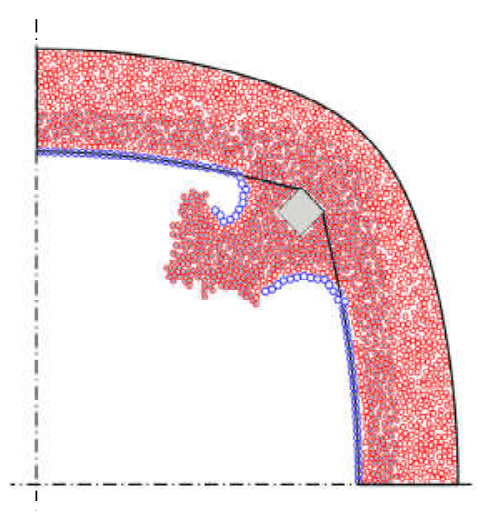

B)

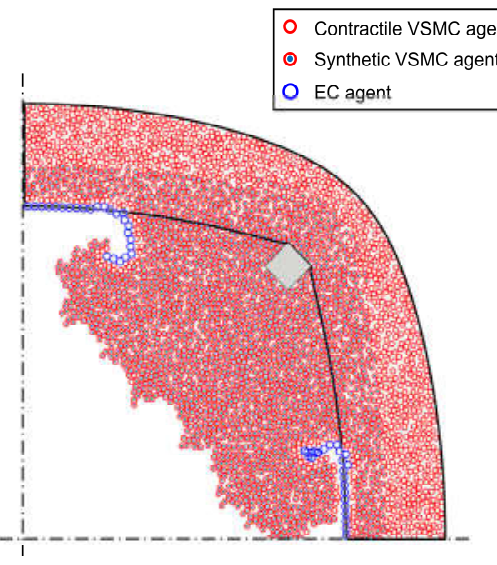

c)

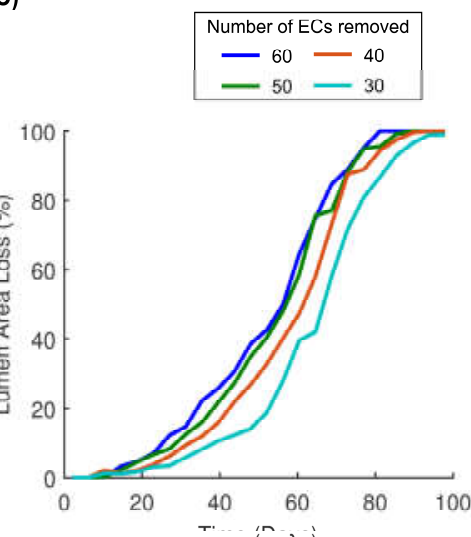

\section{Figure 9}

Result from the $\mathrm{ABM}$ module of the simulation using the $C D M$ showing the predictions at; A) 58 days with a total of 30 EC agents removed, and B) 58 days with $60 \mathrm{EC}$ agents removed. C) Plot of the predicted lumen area loss over time as a function of the number of $\mathrm{EC}$ agents that are removed from the lumen. 


\section{Discussion}

The stent simulation using the IDM results in predictions of in-stent restenosis patterns that are similar to those observed clinically and over a similar timeframe [9]. The inhomogeneous stress distribution and high mean stresses in the CDM induce over-zealous cellular proliferation and consequently high lumen loss, as there is no stimulus to halt cell proliferation. Whilst the presence of ECs in the IDM aids in the control of VSMC proliferation, the VSMCs will cease to proliferate with time (recall Figure 7A) given that damage is progressively removed in the tissue from the model by MMPs.

The failure of the CDM to halt in a realistic timeframe is due to the fact that damage never decreases in the model and in the highest load case, damage continues to increase to the maximum value of 1, see Figure 10A. Even with the inclusion of ECs, the CDM fails to selfregulate and all EC scenarios tested result in full occlusion of the vessel by 100 days, see Figure

9. The growth patterns with ECs present is also clearly non-physiological and does not correlate well with growth patterns observed clinically [20,32]. A physiological response is predicted by the CDM in balloon angioplasty because there is only one cycle of high stress, and damage decreases thereafter, see Figure 10B. 
A)

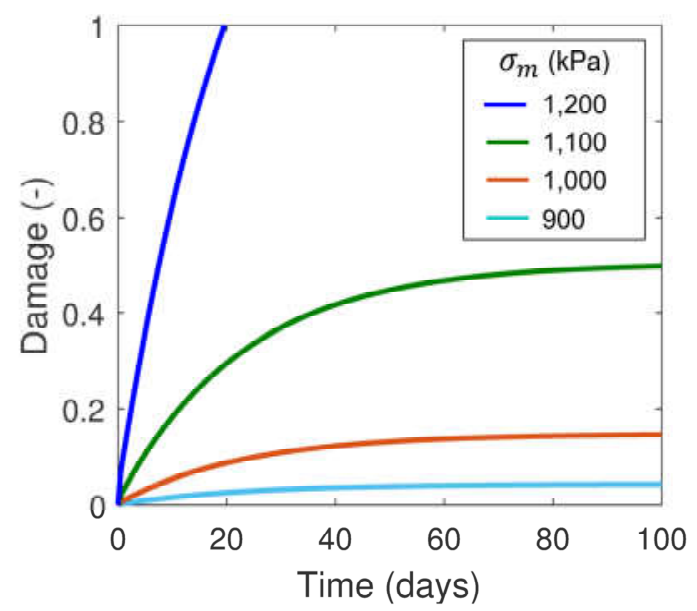

B)

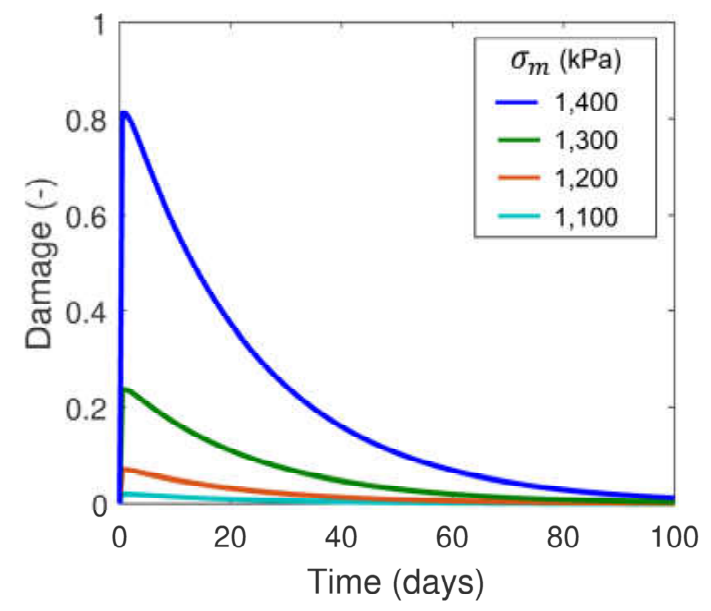

Figure 10

A) Plot of damage with time for the CDM for just one cycle for a range of mean stresses $\sigma_{m}$. Note that the damage decays with time for all loads. B) Plot of damage with time for the CDM at a frequency of $1 \mathrm{~Hz}$ for a range of mean stresses $\sigma_{m}$. Note that in this scenario the damage never decreases and in the highest load case increases all the way to a value of 1 . The probability of proliferation $P_{D T}$ for the CDM is tied to the damage, hence if there is high damage, there is a high probability of VSMC agent proliferation.

In contrast, where initial damage mediates the restenosis process in the IDM model, ECs play a key role in regulating the predicted timeframe of the in-stent restenosis process, whereby removal of 30 ECs by the stent results in a self-limiting process after 36 days and a mere 3\% lumen loss. This contrasts with the model where 60 ECs are removed, which takes approximately 85 days to reach a steady state with $12.5 \%$ lumen area stenosis, see Figure 8 . Without any EC agents, the restenosis growth in the model terminates at $26 \%$ lumen loss.

The mechanobiological framework presented here can readily be adapted to investigate the degree to which EC denudation during stenting influences the restenosis response. Previous studies have demonstrated that remnant patches of ECs post-stenting can reduce neointimal thickness and accelerate the healing process [13], and that the quantity of remnant endothelium is dictated by stent geometry [33]. Rogers et al [34] has shown that a semi-compliant balloon can denude the vessel between the stent struts and this would clearly have a marked influence 
on the restenosis growth patterns post-stenting. Another experimental study investigating the origin of ECs in the healed region post-stenting revealed that these cells proliferated from healthy endothelium proximal and distal to the stent, and originated from bone marrow derived cells [35]. A computational study by Tahir et al. [36] modelled two mechanisms of reendothelialisation; growth from proximal and distal ends and random seeding in the denuded region. Results from these simulations showed that different patterns of neointimal growth and area are predicted by these two mechanisms. The experimental results from [35] imply that both mechanisms occur simultaneously. The 2-D circumferential geometry used in the current study does not allow the simulation of proximal-distal re-endothelialisation, however random seeding may be investigated using this framework.

As demonstrated here, this coupled ABM-FE model is also well designed for investigating different damage mechanisms but it is not limited to the IDM and CDM. Stretch [25], cyclic strain [15,23] and shear stress imposed by blood flow [24,37] are just a few of the other mechanical stimuli that have been postulated to play a role in driving the in-stent restenosis process and this model could be adapted to include such stimuli. It can also be used to explore cell migration along with cell proliferation, or even cell differentiation. Whilst the proliferative capacity of the cells is critical in the progression of in-stent restenosis, the role of different cell origins in the restenosis process may be even more important, given that different cell groups are likely to have varied migratory and or proliferative capacities. Using a cellular Potts model, Tahir et al. [38] have recently presented a model which highlights the importance of VSMC migration and the role that injury induced fenestrae in the intima have on the ability of VSMCs to migrate into the vessel lumen and subsequently proliferate to produce a neointimal hyperplasia. 
In addition, resident stem cells have recently been identified in the media of large arteries which have been postulated to play a highly significant role in instigating the cascade of cellular events that lead to the restenosis post-stenting [39]. Models such as that proposed here provide a means to investigate the likelihood of stem cells regulating this mechanobiological process, however the main limitation to implementing such models is the paucity of empirical data available in the literature which can define the cellular processes in such models and offer a means to validate the outcomes from such models.

Whilst this paper demonstrates that the ABM-FE formulation used in the simulations can successfully capture the key characteristics of in-stent restenosis, there are some limitations to the models. The coupled equations controlling the VSMCs are phenomenological and are therefore sensitive to their constants. It is therefore critical to calibrate these constants. Whilst the calibration using the balloon angioplasty model provides a means to do this, it is very vessel specific and is based on very limited data. Future models will incorporate more mechanistic damage - ECM dynamics, supported by well-designed cell experiments that yield more relevant empirical data. The current framework is also $2 \mathrm{D}$ and not fully coupled; as new geometry is computed by the ABM, the FE model should be re-run with this updated geometry. This will result in lower stresses being experienced by the VSMC agents, attenuating the damage response. This may have an important effect on the resultant behaviour of the CDM. Additionally, residual stresses which exist in the artery wall at a zero pressure state should be included in future FE models [40]. Finally, both VSMCs and ECs are idealised and represented by circles, despite the fact that ECs are squamous epithelial cells and VSMCs are more spindle like in form, particularly contractile VSMCs [41,42]. VSMC density should be more accurately measured using a 3-D microscopy technique such as confocal microscopy or second harmonic generation microscopy. 
These limitations will be further addressed in future work, along with a full investigation of the role of stent design parameters such as strut thickness and the number of struts per cross section. These parameters are known to considerably affect load induced damage in the artery and consequently the degree of in-stent restenosis post stenting [20,21]. This will enable a thorough assessment of the efficacy of different stent designs to achieve sufficient luminal gain, without inducing levels of damage that ultimately result in restenosis in the longer-term.

In the future, it is anticipated that mechanobiological models, such as the ABM-FE framework presented here, will be advanced to include a complex, fibre-based anisotropic constitutive model for the artery, which is capable of remodelling in response to mechanical stimuli such as stress and strain $[43,44]$. It is well known that the fibre alignment of load bearing collagen architecture, in particular, may be critical in terms of health and disease [45] and that fibre architecture has a significant impact on vascular cell growth and differentiation [46]. Ultimately, advances to this mechanobiological framework may enable in silico clinical trials of medical devices to be conducted, where patient specific stochastic models are used to inform the development of more individualised vascular therapies and devices [47].

It is worth noting, however, that all models, regardless of their complexity, structure and/or methodology, are merely an approximation of the physical problem they represent. The ultimate measure of a model's suitability is how well it can describe, or predict, what is physically reasonable, and empirically or clinically observed [48]. To further develop such models and ensure their clinical relevance therefore, requires robust experimental data to define and validate them, and at present there is still a lack of availability of such data. 


\section{Conclusion}

This paper investigated the mechanism behind damage-induced cell proliferation using a coupled finite element and agent based model, assuming it to be driven by a) instantaneous loading, or b) cyclic loading. Furthermore the role of remnant endothelial cells in attenuating in-stent restenosis was explored. Results show that a cyclic damage model predicts a nonphysiological, overly proliferative response. In contrast, the instantaneous model predicts a physiologically realistic growth pattern, is self-limiting within a realistic timeframe, and points to a significant role of endothelial denudation during stent deployment and reendothelialisation in the degree of lumen loss post-stenting. 


\section{Acknowledgements}

This research is funded by Science Foundation Ireland under grant number: SFI 13/CDA/2145.

Computational infrastructure is supported by the Trinity Centre for High Performance

Computing. The authors wish to thank Prof. Jeffrey W. Holmes for generously sharing his agent based model code with us.

\section{References}

[1] M. Heron, Deaths: Leading Causes for 2014., Natl. Vital Stat. Rep. 65 (2016) 1-96. http://www.ncbi.nlm.nih.gov/pubmed/27376998.

[2] M. Nichols, N. Townsend, R. Luengo-Fernandez, J. Leal, A. Gray, P. Scarborough, M. Rayner, European cardiovascular disease statistics 2012, 2012. doi:978-2-9537898-1-2.

[3] L. Roger, A.S. Go, D.M. Lloyd-jones, R.J. Adams, J.D. Berry, T.M. Brown, M.R. Carnethon, S. Dai, G. De Simone, E.S. Ford, C.S. Fox, H.J. Fullerton, C. Gillespie, K.J. Greenlund, S.M. Hailpern, J.A. Heit, P.M. Ho, V.J. Howard, B.M. Kissela, S.J. Kittner, D.T. Lackland, J.H. Lichtman, L.D. Lisabeth, D.M. Makuc, G.M. Marcus, A. Marelli, D.B. Matchar, M.M. Mcdermott, J.B. Meigs, C.S. Moy, D. Mozaffarian, M.E. Mussolino, G. Nichol, N.P. Paynter, W.D. Rosamond, P.D. Sorlie, R.S. Stafford, T.N. Turan, M.B. Turner, N.D. Wong, J. Wylie-rosett, A. Heart, Heart Disease and Stroke Statistics - 2011 Update A Report From the American Heart Association, Circulation. (2011). doi:10.1161/CIR.0b013e3182009701.

[4] R. Kornowski, M.K. Hong, F.O. Tio, O. Bramwell, H. Wu, M.B. Leon, In-Stent Restenosis : Contributions of Inflammatory Responses and Arterial Injury to Neointimal Hyperplasia, J. Am. Coll. Cardiol. 31 (1998) 224-231.

[5] F.G.P. Welt, C. Rogers, Inflammation and restenosis in the stent era, Arterioscler. Thromb. Vasc. $\quad$ Biol. 22 (2002) 1769-1776. doi:10.1161/01.ATV.0000037100.44766.5B.

[6] J. Gunn, N. Arnold, K.H. Chan, L. Shepherd, D.C. Cumberland, D.C. Crossman, Coronary artery stretch versus deep injury in the development of in-stent neointima., Heart. 88 (2002) 401-5. doi:10.1136/heart.88.4.401.

[7] S.-H. Kang, K.W. Park, D.-Y. Kang, W. Lim, K.T. Park, J. Han, H. Kang, B. Koo, B. Oh, Y. Park, D.E. Kandzari, D.J. Cohen, S. Hwang, H. Kim, Biodegradable-polymer drug-eluting stents vs. bare metal stents vs. durable-polymer drug-eluting stents: a systematic review and Bayesian approach network meta-analysis., Eur. Heart J. 35 (2014) 1147-58. doi:10.1093/eurheartj/eht570.

[8] G.N. Kouvelos, N. Patelis, G.A. Antoniou, A. Lazaris, M.I. Matsagkas, Meta-analysis of the Effect of Stent Design on 30-Day Outcome After Carotid Artery Stenting, (2015). doi:10.1177/1526602815598753.

[9] R.S. Schwartz, D.R. Holmes, Pigs, Dogs, Baboons, and Man: Lessons for Stenting from Animal Studies, J. Interv. Cardiol. 7 (1994) 355-368. doi:10.1111/j.15408183.1994.tb00469.x.

[10] J. Thyberg, K. Blomgren, J. Roy, P.K. Tran, U. Hedin, Phenotypic Modulation of Smooth Muscle Cells after Arterial Injury Is Associated with Changes in the Distribution 
of Laminin and Fibronectin, 45 (1997) 837-846.

[11] A.C. Newby, Dual Role of Matrix Metalloproteinases ( Matrixins ) in Intimal Thickening and Atherosclerotic Plaque Rupture, Physiol. Rev. 85 (2005) 1-31. doi:10.1152/physrev.00048.2003.

[12] K. Asanuma, R. Magid, C. Johnson, R.M. Nerem, Z.S. Galis, Uniaxial strain regulates matrix-degrading enzymes produced by human vascular smooth muscle cells, Am. J. Physiol. -- Hear. Circ. Physiol. 284 (2003) H1778--H1784.

[13] C. Rogers, S. Parikh, P. Seifert, E.R. Edelman, Endogenous cell seeding. Remnant endothelium after stenting enhances vascular repair, Circulation. 94 (1996) 2909-2914. doi:10.1161/01.CIR.94.11.2909.

[14] H. Zahedmanesh, H. Van Oosterwyck, C. Lally, A multi-scale mechanobiological model of in-stent restenosis: deciphering the role of matrix metalloproteinase and extracellular matrix changes., Comput. Methods Biomech. Biomed. Engin. 17 (2014) 813-28. doi:10.1080/10255842.2012.716830.

[15] H. Zahedmanesh, C. Lally, A multiscale mechanobiological modelling framework using agent-based models and finite element analysis: Application to vascular tissue engineering, Biomech. Model. Mechanobiol. 11 (2012) 363-377. doi:10.1007/s10237011-0316-0.

[16] C.J. Boyle, A.B. Lennon, M. Early, D.J. Kelly, C. Lally, P.J. Prendergast, Computational simulation methodologies for mechanobiological modelling: a cell-centred approach to neointima development in stents, Phil. Trans. R. Soc. A. 368 (2010) 2919-2935. doi:10.1098/rsta.2010.0071.

[17] A.G. Hoekstra, S. Alowayyed, E. Lorenz, N. Melnikova, L. Mountrakis, B. Van Rooij, A. Svitenkov, G. Závodszky, P. Zun, Towards the virtual artery: a multiscale model for vascular physiology at the physics - chemistry - biology interface, Phil. Trans. R. Soc. A. 374 (2016). doi:/10.1098/rsta.2016.0146.

[18] M. Garbey, M. Rahman, S. Berceli, A multiscale computational framework to understand vascular adaptation, J. Comput. Sci. $8 \quad$ (2015) 32-47. doi:10.1016/j.jocs.2015.02.002.

[19] C.J. Boyle, A.B. Lennon, P.J. Prendergast, In Silico Prediction of the Mechanobiological Response of Arterial Tissue: Application to Angioplasty and Stenting, J. Biomech. Eng. 133 (2011) 81001. doi:10.1115/1.4004492.

[20] R.S. Schwartz, A. Chu, W.D. Edwards, S.S. Srivatsa, R.D. Simari, J.M. Isner, D.R. Holmes, A proliferation analysis of arterial neointimal hyperplasia: Lessons for antiproliferative restenosis therapies, Int. J. Cardiol. 53 (1996) 71-80. doi:10.1016/0167-5273(95)02499-9.

[21] W.H.E. Waale, H. Frederik, H.J.J. Wellens, Modulation of Healthy Pig Coronary Arteries by Self-Expanding Stents, 9 (1996).

[22] A.D. Amore, J.S. Soares, J.A. Stella, W. Zhang, N.J. Amoroso, J.E. Mayer, W.R. Wagner, M.S. Sacks, Large strain stimulation promotes extracellular matrix production and stiffness in an elastomeric scaffold model, J. Mech. Behav. Biomed. Mater. 62 (2016) 619-635. doi:10.1016/j.jmbbm.2016.05.005.

[23] A. Colombo, S. Guha, J.N. Mackle, P.A. Cahill, C. Lally, Cyclic strain amplitude dictates the growth response of vascular smooth muscle cells in vitro: Role in in-stent restenosis and inhibition with a sirolimus drug-eluting stent, Biomech. Model. Mechanobiol. 12 (2013) 671-683. doi:10.1007/s10237-012-0433-4.

[24] A. Caiazzo, D. Evans, J.L. Falcone, J. Hegewald, E. Lorenz, B. Stahl, D. Wang, J. Bernsdorf, B. Chopard, J. Gunn, R. Hose, M. Krafczyk, P. Lawford, R. Smallwood, D. Walker, A.G. Hoekstra, A Complex Automata approach for in-stent restenosis: Twodimensional multiscale modelling and simulations, J. Comput. Sci. 2 (2011) 9-17. 
doi:10.1016/j.jocs.2010.09.002.

[25] B.C. Thorne, H.N. Hayenga, J.D. Humphrey, S.M. Peirce, Toward a multi-scale computational model of arterial adaptation in hypertension: Verification of a multi-cell agent-based model, Front. Physiol. MAY (2011) 1-12. doi:10.3389/fphys.2011.00020.

[26] G.A. Holzapfel, G. Sommer, C.T. Gasser, P. Regitnig, A. Gerhard, G. Sommer, C.T. Gasser, P. Regitnig, Determination of layer-specific mechanical properties of human coronary arteries with nonatherosclerotic intimal thickening and related constitutive modeling, Am. J. Physiol. -- Hear. Circ. Physiol. (2005) 2048-2058. doi:10.1152/ajpheart.00934.2004.

[27] S.M. Peirce, E.J. Van Gieson, T.C. Skalak, Multicellular simulation predicts microvascular patterning and in silico tissue assembly., FASEB J. 18 (2004) 731-733. doi:10.1096/fj.03-0933fje.

[28] S.M. Peirce, E.J. Van Gieson, T.C. Skalak, Multicellular simulation predicts microvascular patterning and in silico tissue assembly., FASEB J. 18 (2004) 731-733. doi:10.1096/fj.03-0933fje.

[29] M. Matsusaki, S. Amemori, K. Kadowaki, M. Akashi, Quantitative 3D Analysis of Nitric Oxide Diffusion in a 3D Artery Model Using Sensor Particles, Angew. Chemie Int. Ed. (2011).

[30] Y.S. Kim, Z.S. Galis, A. Rachev, H.-C. Han, R.P. Vito, Matrix Metalloproteinase-2 and -9 Are Associated with High Stresses Predicted Using a Nonlinear Heterogeneous Model of Arteries, J. Biomed. Eng. 131 (2009) 11009-11010. doi:10.1115/1.3005163.

[31] M.S. Hahn, M.K. McHale, E. Wang, R.H. Schmedlen, J.L. West, Physiologic pulsatile flow bioreactor conditioning of poly(ethylene glycol)-based tissue engineered vascular grafts, Ann. Biomed. Eng. 35 (2007) 190-200. doi:10.1007/s10439-006-9099-3.

[32] G. Nakazawa, M. Vorpahl, A. V. Finn, J. Narula, R. Virmani, One Step Forward and Two Steps Back With Drug-Eluting-Stents. From Preventing Restenosis to Causing Late Thrombosis and Nouveau Atherosclerosis, JACC Cardiovasc. Imaging. 2 (2009) 625628. doi:10.1016/j.jcmg.2009.01.011.

[33] J. Harnek, E. Zoucas, E. Carlemalm, W. Cwikiel, Differences in endothelial injury after balloon angioplasty, insertion of balloon-expanded stents or release of self-expanding stents: An electron microscopic experimental study, Cardiovasc. Intervent. Radiol. 22 (1999) 56-61. doi:10.1007/s002709900329.

[34] C. Rogers, D.Y. Tseng, J.C. Squire, E.R. Edelman, Balloon-Artery Interactions During Stent Placement: A Finite Element Analysis Approach to Pressure, Compliance, and Stent Design as Contributors to Vascular Injury, Circ Res. 84 (1999) 378-383. doi:10.1161/01.RES.84.4.378.

[35] G. Douglas, E. Van Kampen, A.B. Hale, E. McNeill, J. Patel, M.J. Crabtree, Z. Ali, R.A. Hoerr, N.J. Alp, K.M. Channon, Endothelial cell repopulation after stenting determines in-stent neointima formation: Effects of bare-metal vs. drug-eluting stents and genetic endothelial cell modification, Eur. Heart J. 34 (2013) 3378-3388. doi:10.1093/eurheartj/ehs240.

[36] H. Tahir, C. Bona-Casas, A.J. Narracott, J. Iqbal, J. Gunn, P. Lawford, A.G. Hoekstra, Endothelial repair process and its relevance to longitudinal neointimal tissue patterns: comparing histology with in silico modelling., J. R. Soc. Interface. 11 (2014) 20140022. doi:10.1098/rsif.2014.0022.

[37] H. Tahir, C. Bona-Casas, A.G. Hoekstra, Modelling the Effect of a Functional Endothelium on the Development of In-Stent Restenosis, PLoS One. 8 (2013). doi:10.1371/journal.pone.0066138.

[38] H. Tahir, I. Niculescu, C. Bona-Casas, R.M.H. Merks, A.G. Hoekstra, An in silico study on the role of smooth muscle cell migration in neointimal formation after coronary 
stenting, J. R. Soc. Interface. 12 (2015) 20150358. doi:10.1098/rsif.2015.0358.

[39] Z. Tang, A. Wang, F. Yuan, Z. Yan, B. Liu, J.S. Chu, J.A. Helms, S. Li, Differentiation of multipotent vascular stem cells contributes to vascular diseases, Nat. Commun. (2012). doi:10.1038/ncomms1867.

[40] D.R. Nolan, J.P. McGarry, On the Compressibility of Arterial Tissue, Ann. Biomed. Eng. (2015). doi:10.1007/s10439-015-1417-1.

[41] P.W. Alford, A.P. Nesmith, J.N. Seywerd, A. Grosberg, K.K. Parker, Vascular smooth muscle contractility depends on cell shape, 3 (2011). doi:10.1039/c1ib00061f.

[42] R.G. Thakar, Q. Cheng, S. Patel, J. Chu, M. Nasir, D. Liepmann, K. Komvopoulos, S. Li, Cell-shape regulation of smooth muscle cell proliferation, Biophys. J. 96 (2009) 3423-3432. doi:10.1016/j.bpj.2008.11.074.

[43] A. Creane, E. Maher, S. Sultan, N. Hynes, D.J. Kelly, C. Lally, A remodelling metric for angular fibre distributions and its application to diseased carotid bifurcations, Biomech. Model. Mechanobiol. (2012). doi:10.1007/s10237-011-0358-3.

[44] D.R. Nolan, A.L. Gower, M. Destrade, R.W. Ogden, J.P. McGarry, A robust anisotropic hyperelastic formulation for the modelling of soft tissue, J. Mech. Behav. Biomed. Mater. 39 (2014) 48-60. doi:10.1016/j.jmbbm.2014.06.016.

[45] V. Flamini, C. Kerskens, C. Simms, C. Lally, Fibre orientation of fresh and frozen porcine aorta determined non-invasively using diffusion tensor imaging, Med. Eng. Phys. (2013). doi:10.1016/j.medengphy.2012.08.008.

[46] K. Kurpinski, J. Park, R.G. Thakar, S. Li, Regulation of vascular smooth muscle cells and mesenchymal stem cells by mechanical strain, Mol. Cell. Biomech. 3 (2006).

[47] M. Viceconti, A. Henney, E. Morley-Fletcher, In silico clinical trials : how computer simulation will transform the biomedical industry, Int. J. Clin. Trials. 3 (2016) 37-46.

[48] A. Valentín, J.D. Humphrey, Parameter sensitivity study of a constrained mixture model of arterial growth and remodeling., J. Biomech. Eng. 131 (2009) 101006. doi:10.1115/1.3192144. 


\section{Supplementary Figure}

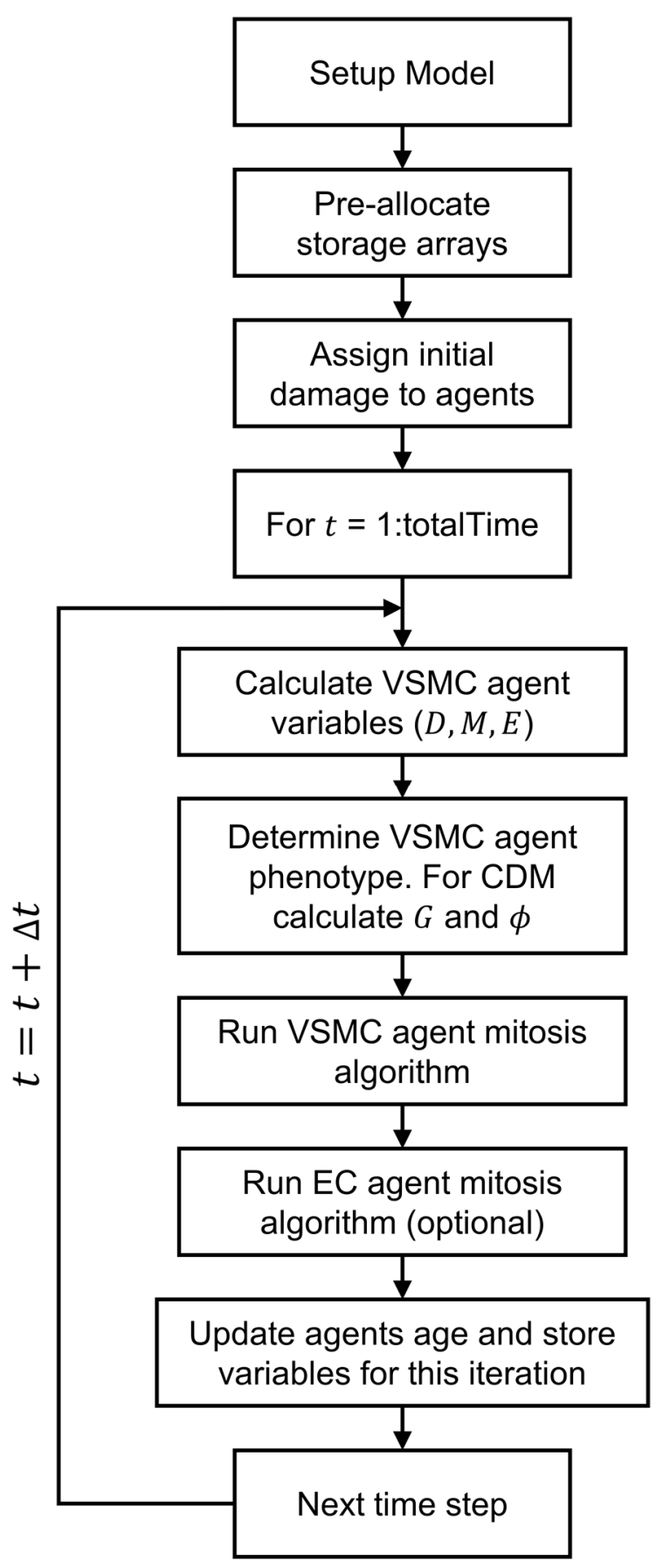

